Proceedings of the

International Geometry Center

Vol. 14, no. 1 (2021) pp. 61-80

\title{
Flows with collective dynamics on a sphere
}

\author{
Alexandr Prishlyak, Andrii Prus, Sophija Huraka
}

\begin{abstract}
In this article different properties of flow codes are studied and a diagram is constructed as a whole topological invariant of them. In particular, flows with at most 6 saddles are described. Two types of simple bifurcations: positive and negative are considered as well. Summarizing the results on compact surfaces with boundary remains an interesting question for future works.
\end{abstract}

Анотація. У даній роботі досліджуються потоки з колективною динамікою на сфері. Сфера розбивається на дві області, в одній з яких Гамільтонова динаміка, а в іншій градієнтна динаміка. Вивчаються різні властивості кодів потоку та будується діаграма як повний топологічний інваріант даних потоків. Зокрема, описані потоки з не більш, ніж 6 сідловими точками. Також розглядаються два типи простих біфуркацій до потоків Морса: позитивні та негативні.

\section{INTRODUCTION}

A smooth function $f$ on an orientable surface equipped with Riemannian metric and a symplectic 2-form generates two vector fields: the gradient field gradf and the skew gradient field sgradf. The trajectories of sgradf are contained in the level-sets of $f$, while the gradient vector field is orthogonal to them. These fields have different structures and dynamics. The corresponding flows are called gradient and Hamiltonian respectively. We will consider dynamical systems in which both such structures are present. By a flow with collective dynamics we will mean a flow having a single or several heteroclinic cycles (which will be called distinguished) that divides the surface into two regions, in one of which there is gradient dynamics, and

2010 Mathematics Subject Classification: 37c10, 37c15, 37c20

UDC 517.956.4

Keywords: flows, collective dynamics, topological invariant, simple bifurcations

DOI: http://dx.doi.org/10.15673/tmgc.v14i1.1902 
in the other, Hamiltonian dynamics. Distinguished cycles are the boundary of each of these areas.

In [3], this and other dynamical systems with collective dynamics were studied under certain conditions on their symmetry and analytical properties. During bifurcation, these conditions can be violated, so it is important to know the possible structures of such systems without the above conditions.

M. Peixoto [9], A. Oshemkov, and V. Sharko [8] and others received a structural classification of Morse-Smale vector fields on closed surfaces [17].

A Morse-Smale flow without closed orbit is called a Morse flow. We say that a flow from some class $X$ of flows on a surface is optimal if it has the least number of fixed points among all flows from $X$. A Morse flow on the closed surface is optimal if and only if it has only one sink and one source [18]. Such a flow is also called a polar Morse flow. The topological structure of polar (optimal) Morse flows on closed surfaces was described in $[4,5,10,18]$. It is convenient to use chord diagrams as complete topological invariants of polar Morse flows. Morse-Smale flows with singularities on a surface with boundary were investigated in [7,12-14]. Since the trajectories of Hamiltonian vector field sgradf are contained in the level sets of $f$, in order to define such vector field (up to topological equivalence) we need to know only the direction on that vector field. In fact, an ordered pair consisting of sgradf vector and a transversal vector corresponding to the direction of increasing of the function uniquely determine an orientation of the Hamiltonian region. A topological classification of Hamiltonian vector fields on surfaces is similar to classifications of functions. Classical papers in this topic are $[2,6,11,15,16]$.

\section{Simple Flows With COLLECTIVE DyNAMiCS}

Simple Morse functions are functions of general position in space of smooth functions (in the sense that they constitute an open and everywhere dense subset). Regular components of level sets of such functions are homeomorphic to circles, while critical components are either points or homeomorphic to figure eight 8.

A flow on a 2-dimensional sphere with a heteroclinic cycle which contains all saddle points will be called simple whenever the following conditions hold:

1) each fixed point of that flow is either non-degenerate or center (see Figure 2.1),

2) no trajectories connect saddles, except for the trajectories of the selected heteroclinic cycle, which divide the surface into two regions, 

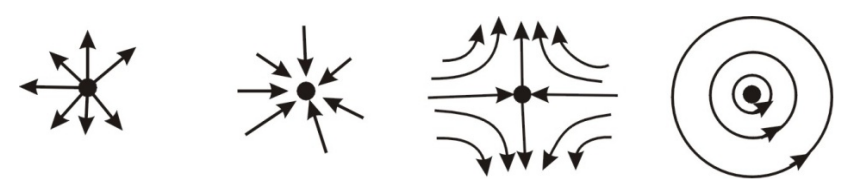

FiguRE 2.1. Fixed points of a simple flow

3) in a region with gradient dynamics (and on its boundary), fixed points have one of three types: sources, sinks, saddles, and the limit set of any trajectory consists of two fixed points,

4) in a region with Hamiltonian dynamics (and on its boundary) there is a fixed point, which is a centre, and all other trajectories are closed.

Thus, the dynamics in the gradient region is the same as for gradient flows of Morse functions.

\section{Diagram of Simple flows on the SPhere}

By definition the region with Hamiltonian-type dynamics of a simple flow is homeomorphic to the open 2-disk and has a centre inside without saddles. We will construct a diagram being a complete topological invariant of such flow. The diagram is a planar graph, which is a circle with some segments drawn inside (Figure 3.1).
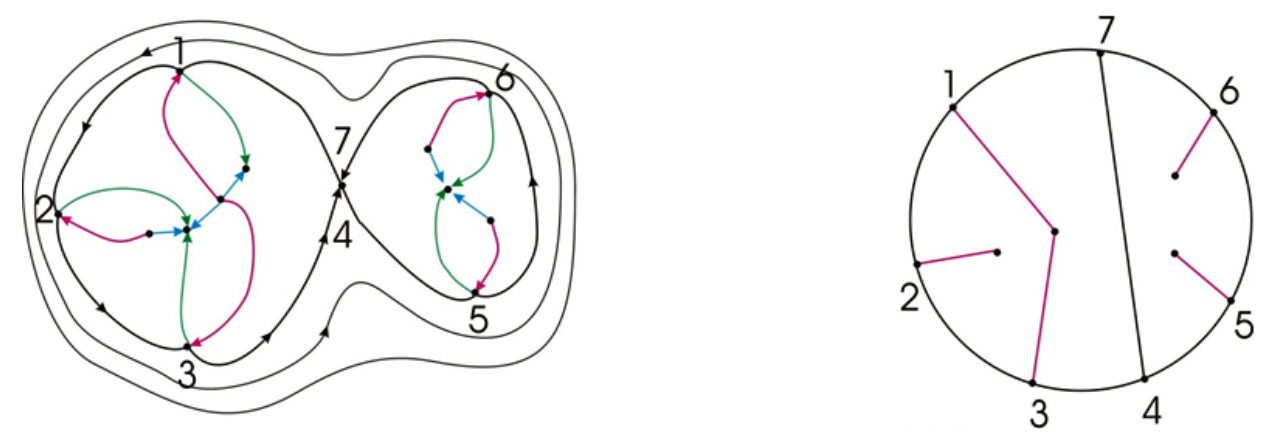

Figure 3.1. Phase portrait of a flow and diagram with code $(1,3),(2),\{4,7\},(5),(6)$

The circle (the distinguished cycle on the graph) corresponds to a closed trajectory in the Hamiltonian region, which is quite close to the distinguished heteroclinic cycle. The vertices correspond to the saddle points and sources, and the saddle lying on the boundary of two connected components of the gradient region corresponds to two vertices. Segments (edges that do not belong to the distinguished cycle) correspond to separatrices coming from a source. Finally, chords connecting vertices correspond to one saddle. 
Thus, a diagram is a graph with a distinguished oriented cycle (oriented counterclockwise in the picture).

Two diagrams are called isomorphic if there is an isomorphism of graphs that maps the distinguished cycle into the distinguished cycle and preserves the orientation of those cycles.

Theorem 3.1. Two simple flows are topologically equivalent if and only if their diagrams are isomorphic.

Proof. Necessity. The restrictions of a homeomorphism inducing a topological equivalence of simple flows to a distinguished cycle and stable manifolds of saddle points yields an isomorphism of the corresponding diagrams.

Sufficiency. An isomorphism of diagrams implies an existence of a homeomorphism, which, while maintaining the direction of motion, maps the selected cycle into a selected one, and stable manifolds of saddle points into stable ones. Such a homeomorphism can be extended to the gradient region is the same way as for Morse flows. To do this, it should be noted that there is a single sink in each area into which the diagram divides the disk (Lemma 3.3). Consider separatrices connecting saddle points on the border with the sinks. Then the bijection between the sinks extends to a unique bijection between the given separatrices. Fix sufficiently small neighborhoods of sources and sinks in which the vector field generating the flows are topologically equivalent either to the vector field $\{x, y\}$ or to $\{-x,-y\}$. Choose Riemannian metrices so that the corresponding separatrices have the same length. On the boundary of neighborhoods of sources those homeomorphisms are fixed (homeomorphisms of circles), which preserve the intersections with the separatrices. These homeomorphisms also define a bijection between the set of trajectories connecting the sources with the sinks, and according to this bijection, the trajectories are mapped to the trajectories proportionally to the lengths of arcs on them. In the same way unstable separatrices are mapped. Together all these homeomorphisms define the needed homeomorphism of all the surface. The continuity of the constructed map follows from the fact that the map preserves the lengths of the trajectories and that the separatrixes have the same length.

To continue to the Hamiltonian region, orthogonal trajectories to the integral curves should be considered in this area. For one orthogonal trajectory further is constructed a homeomorphism of the corresponding trajectory of the second field. Finally, the product of this homeomorphism and the homeomorphism of the selected cycle forms a homeomorphism of the Hamiltonian region (the centre is mapped into the centre). 
Theorem 3.2. A flow diagram has the following properties:

(1) there is an embedding of the graph into a 2-disk which maps the distinguished cycle to the boundary of that disk;

(2) vertices of the distinguished cycle have valency 3 ;

(3) remove edges of the distinguished cycle, then each connected component of the resulting graph will contain at most one vertex that is not included into the distinguished cycle;

(4) there is at least one vertex inside each domain obtained by dividing disk with chord.

Proof. (1) If the complement to the Hamiltonian region is a sphere with a hole (i.e. a 2-disk), then the distinguished cycle is by definition the boundary of that disk.

Otherwise, the complement to the Hamiltonian region is a closed disk in which finitely many pairs of points on the boundary are identified. Such points correspond to the points of self-intersection of the distinguished cycle. Choose a neighborhood of a distinguished cycle in the Hamiltonian region and fix a homeomorphism which maps the distinguished cycle into a given neighborhood. The distinguished cycle (dashed line) in the neighborhood of the sections will be replaced by an embedded circle with chords as in Figure 3.2.

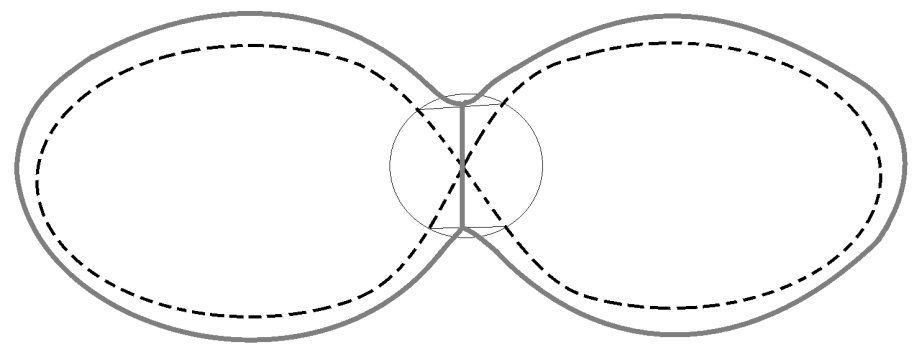

FigURE 3.2.

(2) If the vertex is not a self-intersecting point of the distinguished cycle, then the saddle is incident to three edges that correspond to two separatrices of the distinguished cycle and one stable separatrix. The self-intersecting point of the distinguished cycle corresponds to a chord (edge) and its ends are the vertices of valence 3 .

(3) A graph without a distinguished cycle will consist of stable separatrices. They have no limit points other than sources. Therefore, each component will contain either one vertex being a source, or no vertices if it is a chord corresponding to the self-intersecting points of the distinguished cycle. 
(4) According to the definition of the Morse-Smale flow, there are no sources at the boundary of the area, each trajectory that lies inside the area begins at the source. Therefore, there is at least one vertex inside each area.

Lemma 3.3. There is exactly one sink inside each area, which is obtained by dividing disk by edges of the graph.

Proof. This fact can be explained by calculating the index of the vector field. Consider an example of a polygon with a standard vector field as in Figure 3.3.

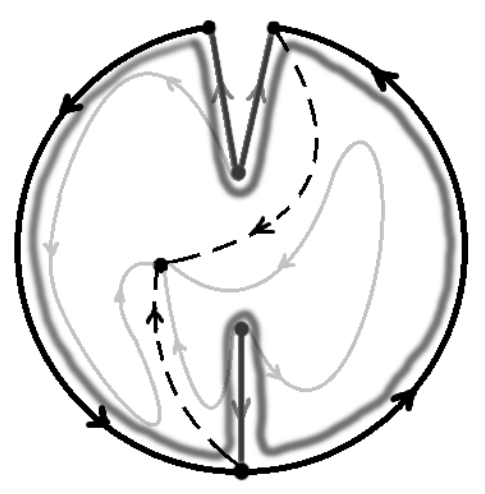

FigURE 3.3.

We calculate the index of the vector field when traversing a trajectory close to the boundary of the lower part of the polygon. For the convenience consider a vector field with a perpendicular separatrix (Figure 3.4): At

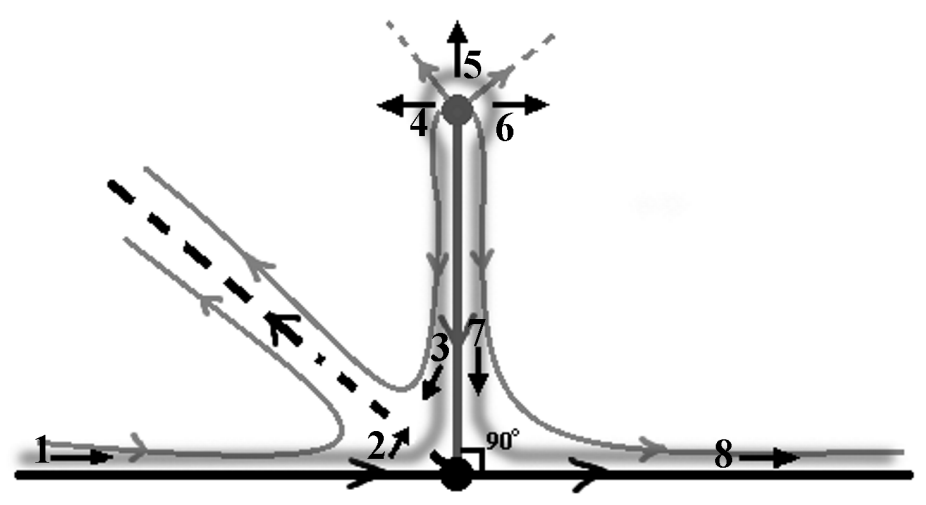

FigURE 3.4 .

point 2 , the vector is rotated by the angle $+45^{\circ}$ (counterclockwise) with respect to the vector 1 . At point 3 by the angle $+180^{\circ}$, at point 4 by the 
angle $-45^{\circ}$, at point 5 by the angle $-90^{\circ}$, at point 6 by the angle $-90^{\circ}$, at point 7 by the angle $-90^{\circ}$ relatively to point 6 , and at point 8 by the angle $+90^{\circ}$. As a result, the vector is rotated by

$$
+45^{\circ}+180^{\circ}-45^{\circ}-90^{\circ}-90^{\circ}-90^{\circ}+90^{\circ}=0^{\circ} .
$$

Therefore, when passing this separatrix, the index of the vector field is not changed. The same can be proved for the upper part of the polygon. Therefore, the sum of indices of all fixed points with respect to the vector field along this polygon is equal to 1 .

Now suppose that the boundary of the polygon is contracted to a point, so we will get a 2-sphere $S^{2}$. By Poincaré-Hopf theorem the sum of indices of all fixed points with respect to the vector field generating the flow is equal to the Euler characteristic of the surface, i.e. 2. On the other hand, the sum of indices of fixed points is equal to 1 , and since each sink has index 1 and there are no other singular points within the region, so there must be only one sink.

Theorem 3.4 (Realization). For each graph with a distinguished oriented cycle satisfying properties (1)-(4) of the previous theorem, there is a simple flow whose diagram is isomorphic to that graph.

Proof. The proof is similar to the proof of the realization theorem in the article [1]. Using the first condition, embed the graph into a 2-disk so that the distinguished cycle is mapped onto the boundary of that 2-disk. Contract to a point each edge whose both ends belong to the distinguished cycle. We cut the disk by an attached graph, and map the resulting connected components into regular polygons with a homeomorphism which is smooth in the interiors of those components, and proportional to the length of the arcs on the edges for a fixed Riemannian metric on the surface. Further we fix a sink point inside each of the obtained polygons. By Lemma 3.3, this sink is unique in each polygon. Fix also a separatrix connecting a source with a saddle and also one of the trajectories connecting the source with the sink in each polygon. Then these polygons are cut into the given trajectories. We will obtain curvilinear triangles or squares.

Fill them with trajectories "parallel" to the chosen trajectory in the boundary. For instance, one can use the following map (Figure 3.5(a))

$$
(x, y) \mapsto(-x(2-x) ; y(2-y)), \quad x \in[0 ; 2], y \in[0 ; 2]
$$

for a triangle, and

$$
(x, y) \mapsto(\sin x \cdot \cos y ; \sin y \cdot(2-y)), \quad x \in[0 ; \pi], y \in[-\pi ; 0] .
$$

for square (Figure 3.5(b)). 


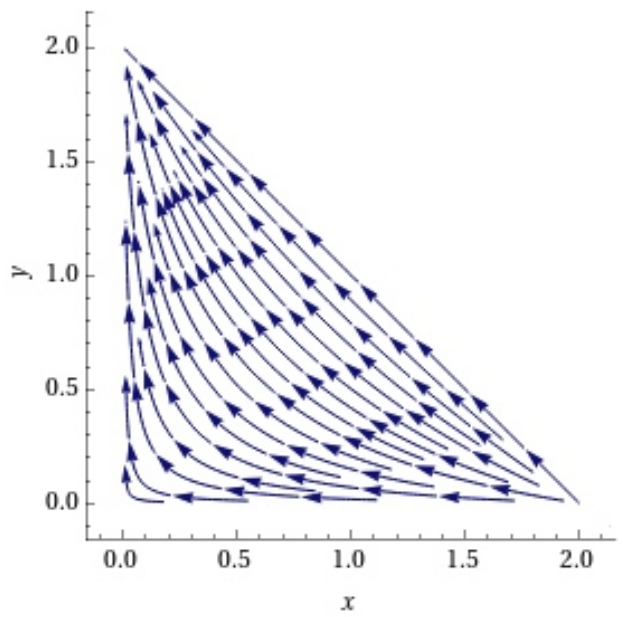

(a)

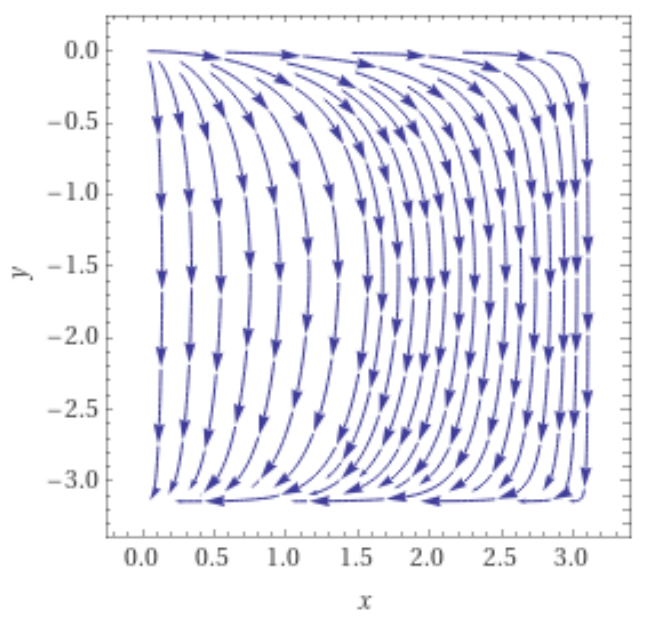

(b)

Figure 3.5.

We fix the neighborhood and define one of the vector fields in it: $\{x ; y\}$ or $\{-x ;-y\}$ for each fixed point. Then boundaries of these neighborhoods are fixed. Another neighborhood, larger than the previous one, is taken. We set the parameterization by a smooth function so that at the boundary of the external neighborhood the velocity vectors have length 1 . Next outside of these neighborhood, the trajectories parameterized so that all velocity vectors are of length 1 . So now the vector fields on both triangles and squares are defined. By gluing polygons along the cuts we get the needed flow in the gradient region. The Hamiltonian region can be mapped onto the 2-dimensional disk, inside of which trajectories are concentric circles. By gluing the Hamiltonian and gradient regions and smoothing the field at the border, we get the required vector field.

\section{FLOW CODE}

By sequentially numbering the vertices on the circle, we divide them into groups and distinguished pairs. One group includes those vertices of the saddle, which possess separatrices from one source (we denote them by $(1,2,3)$ ), and chords corresponding to the number of pairs of vertices (we denote them by $\{1,2\})$.

Definition 4.1. A flow code is a set of lists of integers from 1 to $n$.

Two codes are called equivalent if one of them can be obtained from another one by cycling renumbering, followed by reordering numbers within the lists and the lists among themselves. 
Theorem 4.2. Two flows are topologically equivalent if and only if their codes are equivalent.

Proof. Necessity. By construction, the code is uniquely determined by the saddle number 1 . Arbitrariness in selecting this saddle leads to equivalent codes. Therefore, we can assume that the given topological equivalence maps the first saddle to the first one. Then, taking to account that the distinguished cycle and the direction of movement along it are preserved, this topological equivalence retains the numbers of saddles. Moreover, it transfers separatrices into separatrices and sources into sources. Therefore, the lists that correspond to them will be the same. As a result, the flow codes will be equivalent.

Sufficiency. An equivalence of the codes (saddle numbers) specifies the correspondence between the vertices as well as the correspondence between the edges of the distinguished cycle. The mapping of the first type of the list specifies the mapping between the vertices (sources) and their outgoing edges, and the second type of the list specifies the mapping between the chords. Thus, an equivalence of codes determines a unique isomorphism of diagrams and therefore a topological equivalence of flows.

Using cyclic permutations of numbers in the lists and cyclic permutations of the lists we can assume that the numbers in each list are written in ascending order; the lists are ordered by the value of the first number in the list. Then the following properties of flow code follow from constructions and the latter assumptions:

(1) Each integer appears only once (and thus only in one of the lists). The numbers in each list are written in ascending order; the lists are ordered by the value of the first number in the list.

(2) The lists are divided into two types: the first one, written in parentheses (), can have an arbitrary number of elements; the second one, in braces \{\} , consists of two numbers.

(3) For the lists, the rule of not crossing is fulfilled: for any elements $a, b$ from one list and $c, d$ from another list, we can not have that $a<c<$ $b<d$.

(4) There are no pairs of the second type like $\{a, a+1\}$ or $\{1, n\}$, as well as no sequence of pairs of the second type like

$\left\{a_{1}, a_{2}-1\right\},\left\{a_{2}, a_{3}-1\right\},\left\{a_{3}, a_{4}-1\right\}, \ldots,\left\{a_{k-1}, a_{k}-1\right\},\left\{a_{1}-1, a_{k}\right\}$

or if $a_{1}=1$, then $\left\{a_{k}, n\right\}$ is the last pair in this sequence (Figure 4.1).

Remark 4.3. Geometrically property (3) is equivalent to the fact that chord $a b$ does not cross chord $c d$, while property (4) means that chords of the second type divide the disk into parts, each of which contains at least 


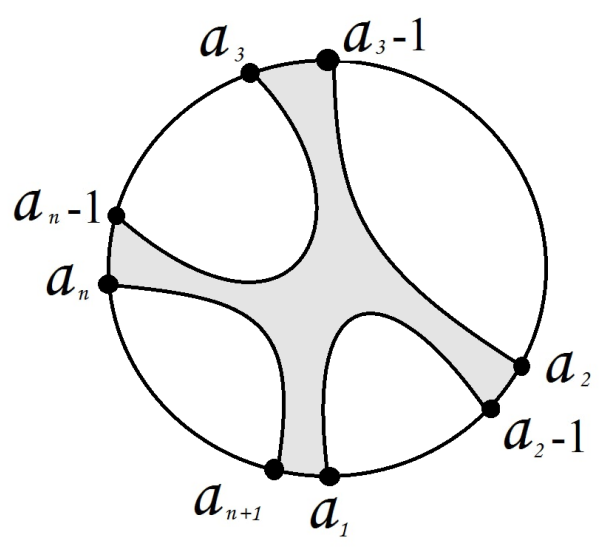

Figure 4.1.

one source and, accordingly, at least one saddle associated with it by a separatrix.

A code that satisfies the conditions (1)-(4) will be called valid.

Theorem 4.4. For each valid code there is a simple flow with such code.

Proof. Given a flow code, we should construct a diagram of a flow. For this, we first define the distinguished cycle with the direction of motion, whose vertices are enumerated sequentially with numbers $1,2, \ldots, n$, where $n$ is the maximal number occurring in the code. We will map each list of the first type to a source that is connected by edges to vertices whose numbers are included in this list. Pairs of vertices in the lists of the second type are connected by edges. This gives a flow diagram. Using the diagram implementation Theorem 3.4, we get the required flow.

We will recall now the construction of four color graph. The separatrices belonging to the heteroclinic cycle are colored in black. The rest of the separatrices entering the saddle are colored in red, and those coming out of saddle in green. In each component into which the separatrices divide the gradient region, one trajectory is chosen (starting at the source and ending in the sink) and that trajectory is colored in blue. Colored trajectories form a graph. A dual graph to this one is constructed with the same colors of the corresponding edges. In this case, the vertices corresponding to the curvilinear triangles are colored black, and the remaining vertices in white. According to [1], a four-color graph constructed in this way is a complete flow invariant.

Let's consider now how to construct a four-color graph according to a code or diagram. From the properties described in [1] it follows that the heteroclinic cycle corresponds to a cycle consisting of red and green edges 
alternating with each other. Moreover, white and black vertices also alternate. Thus each white vertex corresponds to an edge of a heteroclinic cycle, and each black vertex corresponds to a saddle at that point. Black vertices and the corresponding red edges are enumerated in the same way as when constructing the code. The direction of movement along the heteroclinic cycle determines the orientation of the red-green cycle.

Consider the case of one heteroclinic cycle (no parentheses in the code). Since for the optimal flow all saddles are located on the heteroclinic cycle, the red-green cycle contains all the vertices of the graph. To construct the graph, blue and black edges should be drawn. The end of each red edge corresponding to the number in the code is connected with the beginning of the red edge corresponding to the next number. For example, if a code contains a list $(1,3,4)$, then the end of the first red edge is connected with the beginning of the third, the end of the third is connected with the beginning of the fourth, while the end of the fourth edge to the beginning of the first one. Since each number is included in the lists once, each vertex of the graph will be incident to some blue edge. Another black vertex is added and connected by the edges to the white vertices. The cyclic order of the edges in it is determined by the direction of movement along the heteroclinic cycle. If several heteroclinic cycles with common saddles are present (curly braces \{\} in the code), then for each component of the gradient area a fourcolor graph can be constructed as before. In this case, the last added vertex is common to all graphs. The cyclic order of the edges in it is determined by the numbering of the saddles. Thinking in reverse order, a diagram and a code can be built using a 4-colour graph of a optimal flow on a sphere.

Using these invariants, we find in next sections all possible structures of such flows with no more than 6 saddles.

\section{Flows With NO MORE THAN 3 SADDLES}

Theorem 5.1. Up to a topological equivalence there exists

(I) a unique simple flow with one saddle: (1)

(II) two simple flows with two saddles:
1) $(1)(2)$,
2) $(1,2)$.

(III) four simple flows with three saddles:
1) $(1)(2)(3)$;
2) $(1,2)(3)$
3) $(1,2,3)$;
4) (1)(3) $\{2,4\}$.

Their diagrams are shown in Figure 5.1.

Proof. (I) For each source there is at least one separatrix that goes out of it, since otherwise each trajectory from the source would go to the sink which is possible only on a sphere. If there is only one saddle in the flow, 


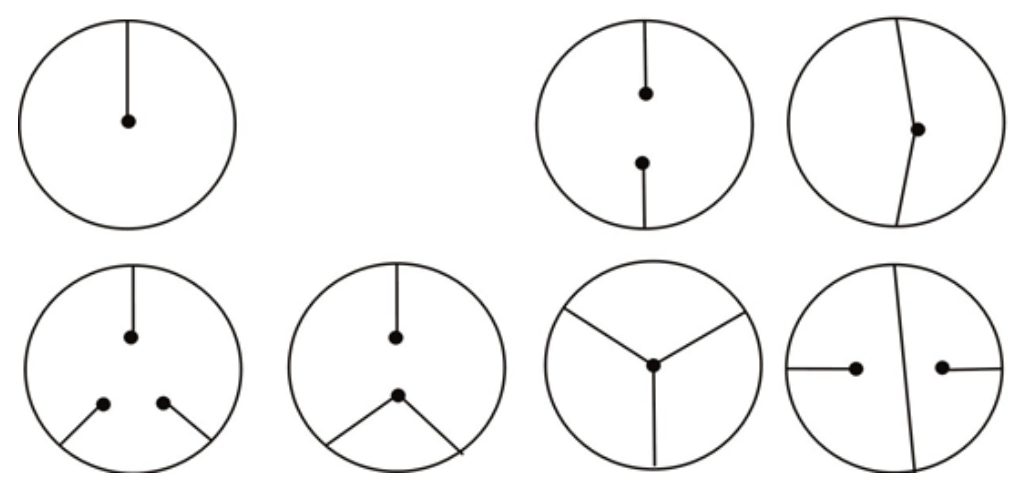

FIgURE 5.1. Diagrams of simple flows with 1,2 and 3 saddles

then the source is unique. Therefore the source must be connected with saddle. By providing this saddle with the number 1, we get the following code: (1). See picture 1 in Figure 5.1.

(II) If a flow has exactly 2 saddles, then there can be either 1 or 2 sources. If there are two sources, then each source is connected to the corresponding saddle, (picture 2). Otherwise, when the source is unique, then two trajectories to each saddle must come out of it, (picture 3). Thus, if the source is the only one, we have the code: $(1,2)$. If there are two sources, then the code is $(1),(2)$.

(III) If there are 3 saddle points in the flow, then there can be at least one and no more than three sources. If there are three sources, then each of them is connected by a trajectory to a separate saddle. In this case, code $(1)(2)(3)$ is obtained, (picture 4). If there are two sources, then one of them is connected by trajectories with two saddles, and the other with the third saddle. Thus, code $(1,2)(3)$ is formed, (picture 5). If the source is the only one, it is connected by three trajectories with three saddles. In this case, the code $(1,2,3)$ is formed, (picture 6 ). Another possibility is when saddle points form two heteroclinic cycles with one intersection point. Then there are only two saddles that can be connected to the source. Thus, the code is formed: (1)(3) $\{2,4\}$, (picture 7 ).

\section{Flows With 4 SADDLES}

Theorem 6.1. There are 8 topologically non-equivalent simple flows with 4 saddle points. They are described by the following codes, see Figure 6.1.
1) $(1)(2)(3)(4)$;
5) $(1,2)(3,4)$;
2) $(1,3)(2)(4)$;
6) $(1,2,3,4)$;
3) $(1,2)(3)(4)$;
7) $(1)\{2,5\}(3)(4)$;
4) $(1,2,3)(4)$;
8) (1) $\{2,5\}(3,4)$. 

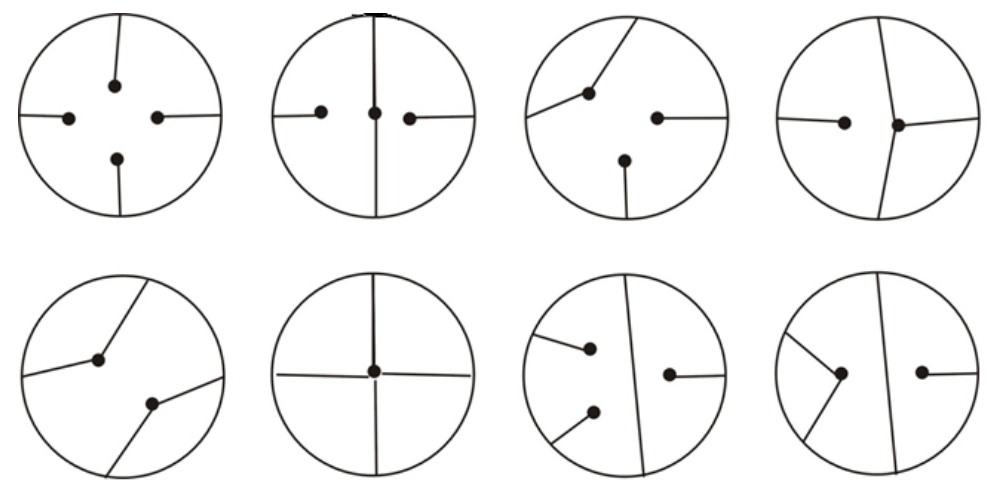

Figure 6.1. Diagrams of flow with 4 saddles

Proof. First consider the case when saddle points form a heteroclinic cycle without intersection points. If there are 4 saddle points, then the number of sources can be not less than 1 and not greater than 4 . If there are 4 sources, each of them is connected to a separate saddle. There are no other cases, since each source is connected to a saddle point by at least one trajectory and each saddle can be connected to only one source. Thus, this diagram corresponds to the code $(1)(2)(3)(4)$, (picture 1 of Figure 6.1).

If there are three sources, then one of them must be connected to two saddles, and each of the other two sources must be connected to one saddle. Two separatrices coming out of one source divide 2-disk into two parts, so two variants are possible:

1) two left flows are situated in one part;

2) they are situated in different parts.

This gives the following codes: $(1,3)(2)(4)$ (picture 2) and $(1,2)(3)(4)$ (picture 3) respectively.

If there are two sources, then two options are possible:

1) one of the sources is connected to three saddles, and the other to one;

2) each of the sources is connected to two saddles.

Option 1) corresponds to the code $(1,2,3)(4)$ (picture 4), while option 1) corresponds to the code: $(1,2)(3,4)$ (picture 5$)$.

If there is one source, it is connected to all the saddles. In this case we have only one diagram and the following code corresponds to it: $(1,2,3,4)$ (picture 6).

Consider the case when saddle points form two heteroclinic cycles with one intersection point. Then only three saddles remain which can be connected to the sources. In each of the areas at least one flow is located, 
and, consequently, at least one saddle. Two possible variants of the diagram correspond to codes $(1)\{2,5\}(3)(4)$ (picture 7 ) and $(1)\{2,5\}(3,4)$ (picture 8).

\section{FlOWS WITH 5 SADDLES}

Theorem 7.1. There are 19 different simple flows with 5 saddle points, see Figure 7.1.

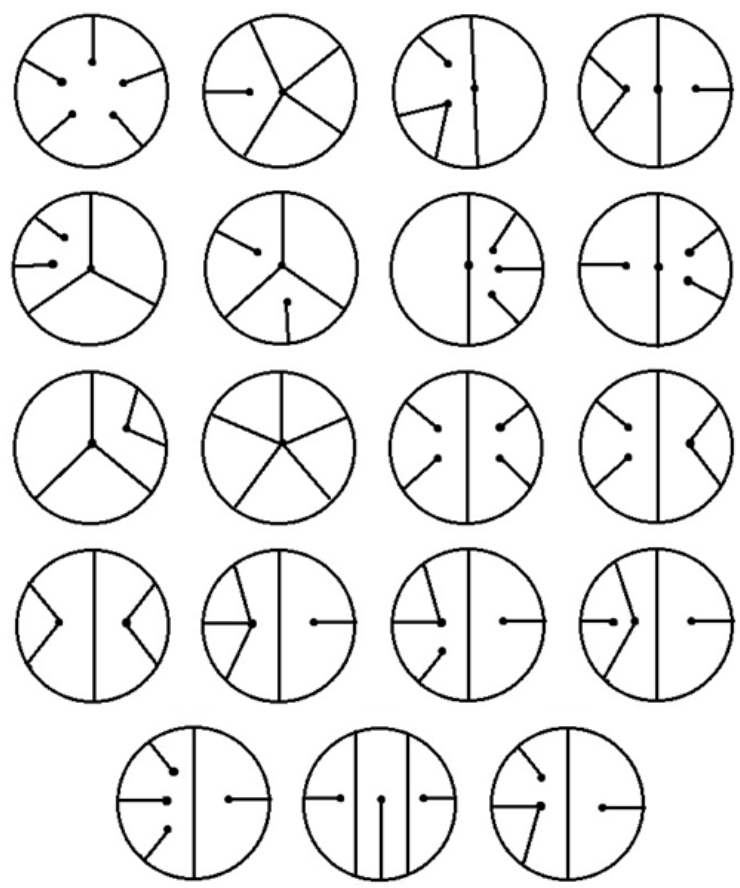

Figure 7.1. Diagrams of flow with 5 saddles

Proof. Consider the flows containing one heteroclinic cycle with 5 saddle points without intersection points. For find all possible diagrams corresponding to these flows we will use representation of the number 5 as a sum of non-negative numbers. Here the number of items is equal to the number of sources, and each item separately is equal to the number of separatrices, coming out from the source. Thus:

- $5=1+1+1+1+1$ : this decomposition corresponds to a diagram with 5 disjoint segments (picture 1);

- $5=4+1$ : this corresponds to a diagram in which 4 segments originate from one point and one segment disjoint from them (picture 2);

- $5=3+2$ : this is a diagram in which 3 segments originate from one point and 2 from another (picture 9); 
- $5=2+2+1$ : here two segments originate from one point, two from another and one disjoint from them. Two pairs of segments coming out of one point divide the disk into three parts. Depending on the location of the last single segment, 2 distinct diagrams are formed (pictures 3 and $4)$;

- $5=3+1+1$ : here three segments from one point and two disjoint segments. Three segments divide the disk into three parts. Depending on the location of the remaining segments, 2 non-homeomorphic diagrams are formed (pictures 5 and 6);

- $5=2+1+1+1$ : here we have two segments originating from one point and three disjoint segments. A pair of segments divide the disk into two parts. Depending on the location of the remaining segments, 2 non-homeomorphic diagrams are formed (pictures 7 and 8);

- $5=5+0$ : here all 5 lines come from the same point. Thus, there are 10 distinct diagrams (picture 10).

- Finally, consider the flows containing two heteroclinic cycles with one point of intersection. Then there are 8 distinct case. There is also one version of the diagram that corresponds to the flow with three heteroclinic cycles with two intersection points.

Thus there are exactly 19 mutually distinct diagrams in total. The codes are of the following form (in the order according to Figure 7.1):
1) $(1)(2)(3)(4)(5)$
11) $(1)(2)\{3,6\}(4)(5)$;
2) $(1)(2,3,4,5)$
12) (1) $(2)\{3,6\}(4,5)$;
3) $(1,2)(3)(4,5)$;
13) $(1,2)\{3,6\}(4,5)$;
4) $(1,2)(3,5)(4)$;
14) $(1,2,3)\{4,6\}(5)$;
5) $(1)(2)(3,4,5)$;
15) $(1,2)(3)\{4,6\}(5)$;
6) $(1)(2,3,5)(4)$;
16) $(1,3)(2)\{4,6\}(5)$;
7) $(1)(2)(3)(4,5)$;
17) $(1)(2)(3)\{4,6\}(5)$;
8) $(1)(2)(3,5)(4)$;
18) (1) $\{2,7\}\{3,5\}(4)(6)$;
9) $(1,2)(3,4,5)$;
19) $(1)(2,3)\{4,6\}(5)$.
10) $(1,2,3,4,5)$;

\section{Flows With 6 SADDLES}

Theorem 8.1. There are exactly 56 topologically nonequivalent simple flows with 6 saddle points.

Proof. Consider the flows with a heteroclinic cycle without intersection points. The corresponding charts are searched using the partition of the number 6 into the sum of non-negative numbers:

- $6=1+1+1+1+1+1$ : this corresponds to a diagram with 6 separate segments; 
- $6=6+0$ : this corresponds all lines come from the same point;

- $6=5+1$ : this corresponds to a diagram in which 5 segments originate from one point and one segment separately;

- $6=4+1+1$ : here four-piece section divides the disk into 4 parts. Depending on the location of the other two lines, there are three different charts.

- $6=4+2$ : here we have exactly one diagram;

- $6=3+3$ : here we have exactly one diagram;

- $6=3+2+1$ : here there are five different diagrams with different line arrangements;

- $6=3+1+1+1$ : this corresponds to four different diagrams with different line arrangements;

- $6=2+2+2$ : here we also have exactly one diagram;

- $6=2+1+1+1+1$ : this corresponds to three different diagrams with different line arrangements;

- $6=2+2+1+1$ : this corresponds to six different diagrams with different line arrangements.

Thus there are 27 diagrams in total. Using the results of counting the number of flows with 2, 3 and 4 saddle points, 21 different flow diagrams were obtained with two heteroclinic cycles with one point of intersection and 8 flow diagrams with three heteroclinic cycles with 2 points of intersection.

\section{Simple Bifurcations to Morse Flows}

Definition 9.1. One-parametric bifurcation of a optimal flow on the sphere is called a simple bifurcation if there is a coordinate system $(x, y)$ in the Hamiltonian region where the field is equal to $|v|\{-y, x\}$ (centre) and is replaced by $|v|\{t x-y, x+t y\}$.

Depending on the sign of $t$, we have to deal with positive or negative bifurcation (source or sink). Our flow code turns into a code similar to the one named after Oshemkov-Sharko (rotation system).

In the gradient region the flow remains topologically equivalent to the initial one. The heteroclinic cycle breaks down into separatrices going to the sources or to the sink.

The Morse flow rotation system (equivalent to the Oshemkov-Sharko code) is a set of lists of natural numbers from 1 to $n$, in which each number occurs twice and corresponds to a saddle. Each list corresponds to a source, while the numbers are written in the order in which separatrices meet when rotating around that source according to the orientation of the surface. Here, each separatrix has the same number as the saddle which it enters. 
Definition 9.2. A positive transformation of code

$$
\left(a_{1}, \ldots, a_{m}\right) \ldots\left(b_{1} \ldots b_{k}\right)\left\{c_{1}, c_{2}\right\}\left\{c_{3}, c_{4}\right\} \ldots\left\{c_{p-1}, c_{p}\right\}
$$

is the replacing it

1) either with the code

$$
\left(a_{1}, a_{1}+1, a_{2}, a_{2}+1, a_{m}, a_{m}+1\right) \ldots\left(b_{1}, b_{1}+1, \ldots, b_{k}, b_{k}+1\right)
$$

if $a_{m}+1, \ldots, b_{k}+1$ are not equal to $c_{i}$, where $j=1, \ldots m ; l=1, \ldots k$.

2) or with the code

$\left(a_{1}, a_{1}+1, a_{2}, a_{2}+1, a_{m}, a_{m}+1, c_{2}+1\right) \ldots\left(b_{1}, b_{1}+1, \ldots, b_{k}, b_{k}+1\right)$

if $a_{m}+1=c_{1}$ and other $a_{m}+1 \ldots b_{k}+1$ are not equal to $c_{i}, c_{2 j}+1 \neq c_{2 j+1}$, and similarly to the other $j$ and lists: if $a_{m}+1=c_{1}$, then after it, another number $c_{2}+1$ is added to this list, if $a_{m}+1=c_{2}$, then $c_{1}+1$ is added; 3) or with the code

$\left(a_{1}, a_{1}+1, a_{2}, a_{2}+1, a_{m}, a_{m}+1, c_{2}+1, c_{4}+1\right) \ldots\left(b_{1}, b_{1}+1, \ldots, b_{k}, b_{k}+1\right)$

if $a_{m}+1=c_{1}$ and other $a_{m}+1 \ldots b_{k}+1$ are not equal to $c_{i}, c_{2}+1=c_{3}$ and other $c_{2 j}+1 \neq c_{2 j+1}$, and similarly to the other situations: if $a_{m}+1=c_{1}$, then after it, another number $c_{2}+1$ is added to this list, $a_{m}+1=c_{2}$, then is added $c_{1}+1$ and similarly for other lists and numbers $c_{i}$, if the added number is $c_{j}$, then the process is repeated;

$A$ negative transformation of the code

$$
\left(a_{1}, \ldots, a_{m}\right) \ldots\left(b_{1} \ldots b_{k}\right)\left\{c_{1}, c_{2}\right\}\left\{c_{3}, c_{4}\right\} \ldots\left\{c_{p-1}, c_{p}\right\}
$$

is the system

$$
\left(a_{1}, \ldots, a_{m}\right) \ldots\left(b_{1} \ldots b_{k}\right)(1, n, n-1, \ldots, 3,2)
$$

followed by replacing $c_{2}$ with $c_{1}, c_{4}$ with $c_{3}, \ldots, c_{p}$ with $c_{p-1}, n+1$ with 1 and possible renumbering of code.

Example 9.3. A code

$$
(1,3,6)(2)(4,5)
$$

has a positive transformation into

$$
(1,2,3,4,6,1)(2,3)(4,5,5,6),
$$

and a negative one into

$$
(1,3,6)(2)(4,5)(1,6,5,4,3,2) \text {. }
$$

Other example are presented after the following theorem.

Theorem 9.4. Under a simple positive (negative) bifurcation an optimal flow on the sphere if transformed to a Morse flow with a rotation system obtained from the flow code by a positive (negative) transformation. 
Proof. The separatrix lying on the heteroclinic cycle between points $a_{1}$ and $a_{1}+1$, after positive bifurcation, will exit the source and lie in a sufficiently small region of combining itself with bifurcation and separatrix coming from source $a_{1}$. This means that in the list of separatrices that come from this source, after a1, separatrix going to $a_{1}+1$ will be added. If also this saddle point is the intersection point of the heteroclinic cycle $\left(c_{i}\right)$, then it should be considered that at the same time we switch from one chord point to another as well as that the next separatrix will also come from this source during bifurcation. By negative bifurcation, all separatrices from the heteroclinic cycle will be transferred to separatrices coming from the newly formed source. Therefore, they, as in this cycle, will be numbered sequentially, but according to the orientation, in reverse order. In this case, the two numbers corresponding to the chord (intersection point of the heteroclinic cycle) must be identified.

Example 9.5. The flow at Figure 3.1 with code

$$
(1,3)(2)\{4,7\}(5)(6)
$$

at simple positive bifurcation will pass into the flow with rotation system

$$
(1,2,3,4,1)(2,3)(5,6)(4,5,6),
$$

and at negative into

$$
(1,3)(2)(5)(6)(1,4,6,5,4,3,2) .
$$

Corollary 9.6. If two simple flows are topologically equivalent, then the flows obtained by simple positive (or negative) bifurcations are also topologically equivalent.

Proof. If simple flows are topologically equivalent, then their codes are equivalent. According to the previous code theorem, the new Morse flow code after bifurcation is unequivocally defined. Therefore, the received codes are equivalent, and thus the obtained Morse flows are topologically equivalent.

Corollary 9.7. If positive bifurcation produces the topologically equivalent flows, then the initial flows are topologically equivalent too.

Proof. It is enough to specify the procedure as the resulting code to restore the source code. For a positive bifurcation, we cross out the last (longest) list. This will give all the lists in the round brackets. If the last list consists of $n$ elements, then we compare it with the list $(1, n, n-1, \ldots, 3,2)$. Let us write out a couple of different elements of these two lists in round brackets. 
So we get all the lists in the round brackets. In this way the source code is restored unequivocally.

Conjecture 9.8. If after negative bifurcation there is a topological equivalence of the obtained flows, which preserves the orientation of the surface, then the initial flows are topologically equivalent (the orientation is consistent with the rotation around the centre).

\section{Conclusion}

The complete topological invariants, a diagram and code flow with collective dynamics on the sphere, in which the Hamiltonian region has the form of a centre, and the gradient is a Morse flow, are built. All possible structures of such flows with no more than 6 points are described. Their simple bifurcations to Morse flows are also analyzed. The problem of describing other possible bifurcations of these flows, as well as summarizing the results on compact surfaces with boundary, still remains a matter of interest.

This article based on the talks given at the Austro-Ukrainian Institute (AUI) seminars (Vienna, Austria) in 2021 and partially supported by the Project between the Austrian Academy of Sciences (AAS) and the National Academy of Sciences of Ukraine (NASU) on Categorical Quantum Theory of Neutron and Photon Optics.

\section{REFERENCES}

[1] Maria Loseva Alexandr Prishlyak. Topology of optimal flows with collective dynamics on closed orientable surfaces. Proc. Int. Geom. Cent., 2020.

[2] A. V. Bolsinov, A. T. Fomenko. Integrable Hamiltonian systems. Geometry, Topology, Classification. A CRC Press Company, Boca Raton London New York Washington, D.C., 2004. 724 p.

[3] O. A. Burylko. Collective dynamics and bifurcations in symmetric networks of phase oscillators. i. Nonlinear Oscillations, 22(2):165-195, 2019, https://www.imath.kiev.ua/ $\sim$ nosc/web/show_article.php?article_id $=1275 \&$ lang $=$ en.

[4] O. A. Giryk. Classification of polar morse-smale vector fields on two-dimensional manifolds. Methods Funct. Anal. Topology, 2(1):23 - 37, 1996.

[5] O.A. Kadubovskyj. Classification of morse-smale vector fields on 2-manifolds. Visn., Mat. Mekh., Kyïv. Univ. Im. Tarasa Shevchenka, (14):85-88, 2005.

[6] A.S. Kronrod. Functions of two variables. Russian Mathematical Surveys, 5:24-134, 1950.

[7] M.M. Loseva, A.O. Prishlyak. The topology of morse-smale flows with singularities on the boundary of 2-dimensinal disk. Proc. Intern. Geom. Center, 9(2):32-41, 2016, doi: $10.15673 /$ tmgc.v9i2.279.

[8] A.A. Oshemkov, V.V. Sharko. Classication of morse-smale flows on two-dimensional manifolds. Matem. Sbornik, 189(8):93-140, 1998. 
[9] M.M. Peixoto. On the classication of flows of 2-manifolds. Dynamical Systems (Proc. Symp. Univ. of Bahia, Salvador, Brasil, 1971), pages 389-419, 1973.

[10] D. Poltavec. Equivalent polar morse-smale systems on two dimensional manifolds of genus 3. In Abstract of International Conference on Topology and its Applications, Kiev, page 29, 1995.

[11] A. O. Prishlyak. Topological equivalence of smooth functions with isolated critical points on a closed surface. Topology Appl., 119(3):257-267, 2002, https://doi.org/10. 1016/S0166-8641(01)00077-3.

[12] A.O. Prishlyak, M.V. Loseva. Optimal morse-smale flows with singularities on the boundary of a surface. J Math Sci, 243:279-286, 2019, doi: 10.1007/s10958-019-045399.

[13] A.O. Prishlyak, A.A. Prus. Three-color graph of the morse flow on a compact surface with boundary. Nonlinear Oscillations, 22(2):250-261, 2019, https://www.imath.kiev. ua/ ${ }^{\sim}$ nosc/web/show_article.php?article_id $=1257 \&$ lang $=$ en.

[14] O. O. Prishlyak, A. A. Prus. Morse-Smale flows on a torus with a hole. Proc. Int. Geom. Cent., 10(1):47-58, 2017.

[15] G. Reeb. Sur les points singuliers d'une forme de pfaff complétement intégrable ou d'une fonction numérique. C.R.A.S. Paris, 222:847-849, 1946.

[16] V.V. Sharko. Functions on manifolds. Algebraic and topological aspects., volume 131 of Translations of Mathematical Monographs. American Mathematical Society, Providence, RI, 1993.

[17] D. S. Malyshev V. E. Kruglov, O. V. Pochinka. A multicolour graph as a complete topological invariant for $\omega$-stable flows without periodic trajectories on surfaces. Sbornik: Mathematics, 209(1):96-121, 2018, doi: 10.1070/SM8797.

[18] R. Shchurko Z. Kibalko, A. Prishlyak. Trajectory equivalence of optimal morse flows on closed surfaces. Proceedings of the International Geometry Center, 11(1):2-26, 18.

Alexandr Prishlyak

Taras Shevchenko National University of Kyiv, Faculty of Mechanics and Mathematics, 4th Academician Glushkov avenue, Kyiv, 03127, Ukraine

Email: prishlyak@yahoo.com

ORCID: orcid.org/orcid.org/0000-0002-7164-807X

Andrii Prus

Taras Shevchenko National University of Kyiv, Faculty of Mechanics and Mathematics, 4th Academician Glushkov avenue, Kyiv, 03127, Ukraine

Email: asp00pr@gmail.com

\section{Sophija Huraka}

Taras Shevchenko National University of Kyiv, Faculty of Mechanics and Mathematics, 4th Academician Glushkov avenue, Kyiv, 02232, Ukraine

Email: sophija_huraka@ukr.net 